\title{
uvby photometry of the CP stars HR 5341, HD 142070, HR 6967, and HR 8434^
}

\author{
Saul J. Adelman \\ Department of Physics, The Citadel, 171 Moultrie Street, Charleston, SC 29409, USA \\ Received 20 November 2000 / Accepted 21 December 2000

\begin{abstract}
Differential Strömgren uvby observations from the Four College Automated Photoelectric Telescope (FCAPT) are presented for the mCP stars HD 142070, HR 6967, and HR 8434 and the CP star HR 5341. The latter star is found to be constant. Improved periods were derived for HD 142070, 3.37189 d, HR 6967, 3.91227 d, and HR 8434, 1.43237 d. Further observations of HD 142070 are needed to phase the magnetic data with the photometry, of HR 6967 to settle minor discrepancies between $y$ and scaled Geneva $V$ photometry, and of HR 8434 to resolve small discrepancies between two uvby photometric data sets.
\end{abstract}

Key words. stars: chemically peculiar - stars: individual: HR 5341 - stars: individual - HD 142070 - stars: individual: HR 6967 - stars: individual: HR 8434

\section{Introduction}

Single-channel differential Strömgren uvby observations of the Chemically Peculiar stars HR 5341, HD 142070, HR 6967, and HR 8434 obtained with the Four College Automated Photoelectric Telescope (FCAPT) are examined. Since September 1996, the FCAPT has operated at Washington Camp, AZ. For each group of variable, check, and comparison stars, the telescope first measures the dark count. Then it observes in each filter the skych-c-v-c-v-c-v-c-ch-sky where sky is a reading of the sky, ch that of the check star, c that of the comparison star, and $v$ that of the variable star. Table 1 summarizes group information (Hoffleit 1982; Hoffleit et al. 1993, SIMBAD database). Corrections were not made for neutral density filter differences among the stars of each group. The comparison and check stars were chosen from supposedly nonvariable stars near the variable on the sky that had similar $V$ magnitudes and $B-V$ colors. Adelman et al. (1998) checked their stability using Hipparcos photometry (ESA 1997). The Scargle periodogram (Scargle 1982; Horne \& Baliunas 1986) and the clean algorithm (Roberts et al. 1987) were used to help find periods.

Send offprint requests to: Saul J. Adelman, e-mail: adelmans@citadel.edu

* Tables 2, 3, 4 and 5 are only available in electronic form at the CDS via anonymous ftp to cdsarc.u-strasbg.fr (130.79.128.5) or via

http://cdsweb.u-strasbg.fr/cgi-bin/qcat?J/A+A/368/225
This paper examines the differential photometry of a metallic-lined (Am) star, a non-magnetic CP star, and three magnetic Chemically Peculiar (mCP) stars. For the Am stars a question of particular importance is whether those outside of the the variability strip on the HR diagram are or are not intrinsically variable. HR 5341 is an Am star found by Adelman (1998) to be possibly variable on the basis of Hipparocs photometry. Both HD 142070 and HR 6967 are relatively poorly studied mCP stars while HR 8434 has been studied more extensively.

As the magnetic CP stars are photometric, spectrum, and magnetic variables, their emergent energy distributions, photospheric abundances, and magnetic field strengths depend upon photospheric location. Since their magnetic and rotational axes are not usually aligned, a distant observer often will see variability as the stars rotate. Hydrodynamical processes including radiative diffusion and gravitational settling in radiative envelopes which have strong magnetic fields are thought to produce their anomalous photospheric abundances which depend on the local magnetic field strength and the time since the star was on the ZAMS (Michaud \& Proffitt 1993 and references therein). FCAPT studies have both improved periods and better defined the shapes of their light curves (see, e.g., Adelman et al. 1999). Using these results astronomers can better relate observations taken at different times, detect variable light curves, and study the period distribution of the mCP stars which yields information on the slowing of their rotational periods. With spectra in addition, surface maps of abundances can be derived. These results serve as tests of the theories to produce their anomalous abundances. 


\section{HD 142070}
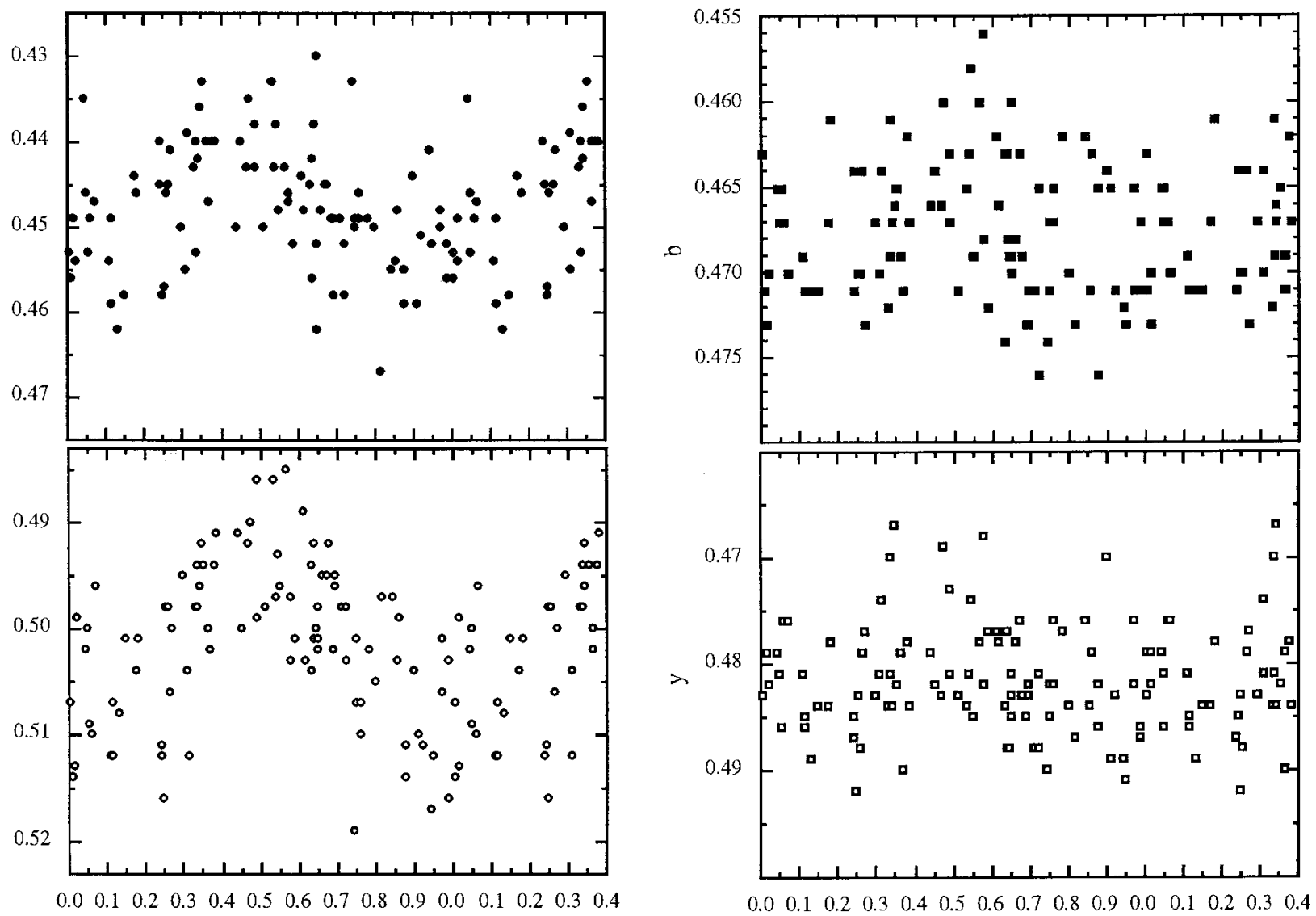

phase

Fig. 1. Differential FCAPT uvby photometry of HD 142070 plotted with the ephemeris HJD $\left(v_{\min }\right)=2450837.499+3.37189 E$

\section{HR 5341}

Adelman (1998) examined the possible variability of the Am stars with Hipparcos photometry and found that as a class they are slightly better candidates for intrinsic phometric constancy than the hotter HgMn stars. As most known variable Am stars are eclipsing binaries, he surmised that many marginally variable Am stars were likely to be members of such systems. Four stars including HR 5341 (HD 124915) were identified for further study. In the 1998-99 and the 1999-2000 observing seasons, 52 and 61 differential uvby observations were made, respectively, with the FCAPT. These should be sufficient to detect any eclipses. Instead these values (Table 2) indicate that HR 5341 was photometrically constant.

\section{HD 142070}

HD $142070\left(\mathrm{BD}-00^{\circ} 3026\right)$ is a relatively poorly studied magnetic CP star. Mathys et al. (1997) found its period to be 3.3748 days and give JD 2449872.600 as the phase of $H_{\text {min }}$. Adelman (1998) noted it was a mCP star whose Hipparcos photometry indicated that it would be worthwhile observing from the ground. During the 199798, 1998-99, and 1999-2000 observing seasons 30, 36, and 26 sets of good differential uvby measurements were made with the FCAPT. The ephemeris which best expresses the variability is HJD $\left(v_{\min }\right)=2450837.499 \pm 0.005+$ $3.37189 \pm 0.00007 \mathrm{E}$. HD 142070 is not a particularly variable star (Fig. 1) with amplitudes of $0.02 \mathrm{mag}$ in $u$, $0.025 \mathrm{mag}$ in $v$, perhaps $0.005 \mathrm{mag}$ in $b$, and $0.01 \mathrm{mag}$ in $y$. The variability appears to be in phase in each bandpass. As the difference in zero points of the magnetic and photometric ephemerides corresponds to 286.16 cycles, it is difficult to relate the magnetic and the photometric results with the currently available data.

\section{HR 6967}

North (1992) used 40 sets of Geneva observations of HR 6967 (HD 171247, BD $+08^{\circ} 3741$ ) to derive a period of 3.9124 days. Catanzaro et al. (1999) confirmed this value using Hipparcos photometry (ESA 1997) and found an error of 0.0004 days. Further He I $\lambda 5876$ shows a sinusoidal variation in equivalent width. Adelman (1998) suggested 
HR 6967
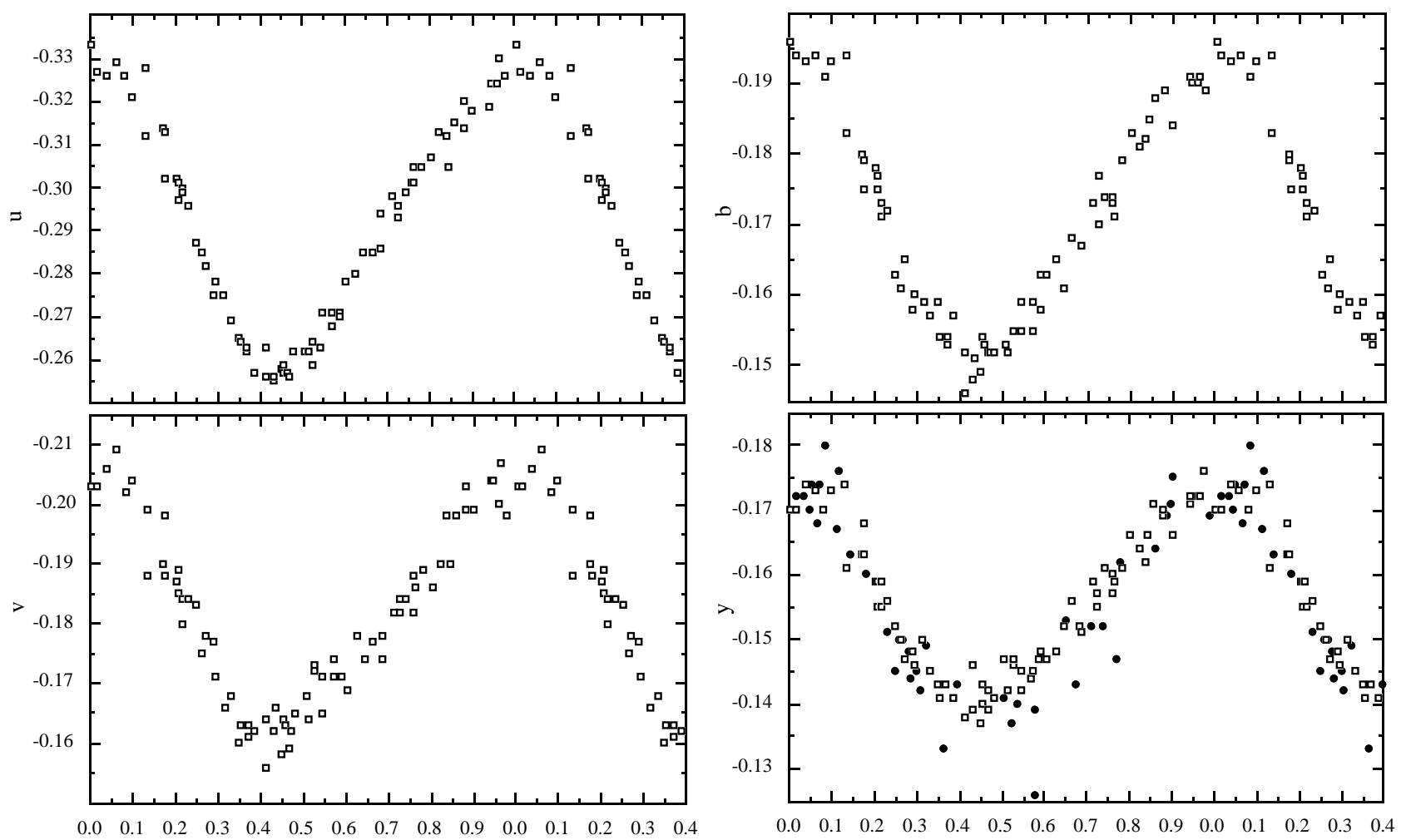

phase

Fig. 2. Photometry of HR 6967 using the ephemeris HJD $\left(y_{\max }\right)=2447179.810+3.91227$ E. The differential Strömgren uvby values of the FCAPT as shown as open boxes while the scaled values from North's Geneva $V$ phomtetry as closed circles

Table 1. Photometric groups

\begin{tabular}{rllll}
\hline HD Number & Star Name & Type & $V$ & Spectral Type \\
\hline 124915 & HR 5341 & v & 6.44 & A9 III \\
124850 & 99 Vir & c & 4.08 & F6 III \\
124553 & HR 5322 & ch & 6.36 & F9 V \\
& & & & \\
142070 & & v & 7.96 & A0 \\
141851 & 36 Ser & c & 5.11 & A3 Vn \\
141378 & HR 5875 & ch & 5.53 & A5 IV \\
& & & & \\
171247 & HR 6967 & v & 6.42 & B8 IIIpSiSr: \\
171975 & HR 6992 & c & 6.42 & B9 V \\
171802 & HR 6985 & ch & 5.39 & F5 III \\
& & & & \\
210071 & HR 8434 & v & 6.39 & A0 III \\
209124 & HR 8389 & c & 6.59 & A0 III-IV \\
211336 & 23 Cep & ch & 4.19 & F0 IV \\
\hline
\end{tabular}

based the space data that this star would be usefully observed from the ground.

In the 1998-99 and 1999-2000 observing seasons 18 and 67 sets, respectively, of good quality differential uvby photometry of HR 6967 were obtained with the FCAPT. An analysis of their period yielded 3.91236 days, essentially the result of North. The Geneva $V$ values with suitable corrections to scale them to the Strömgren $y$ values when both sets of data were plotted with North's ephemeris showed a slight offset. One value with a quality of 1 was not used. To bring the $V$ and $y$ data into agreement required a slight adjustment of the period within the errors set by Hipparcos photometry. Further the phase of $y_{\max }$ was adjusted slightly. The best ephemeris is now HJD $\left(y_{\max }\right)=2447179.810 \pm 0.005+3.91227 \pm 0.00005 E$. Figure 2 shows the variability as a function of phase. The $u, v, b$, and $y$ asymmetric light curves are in phase. The phase of light maximum is slightly uncertain due to the shape of the light curves. Minimum occurs near phase 0.40 . The Geneva $V$ values scatter around the Strömgren $y$ values with a few $m$ mag up to 0.02 mag away from the mean curve. The shape of the minimum might change slightly with bandpass. The amplitudes are $0.075 \mathrm{mag}$ in $u, 0.05 \mathrm{mag}$ in $v, 0.045 \mathrm{mag}$ in $b$, and $0.035 \mathrm{mag}$ in $y$. The asymmetric light curves suggest the surface distribution of the elements is not symmetric about the magnetic poles.

\section{HR 8434}

Adelman et al. (1994) obtained 77 sets of differential FCAPT uvby observations of the magnetic CP star HR 8434 (HD 210071, BD 55²679) over four observing seasons and derived the ephemeris $\operatorname{HJD}\left(U_{\min }\right)=$ $2441613.79+1.43242 E$. 


\section{HR 8434}
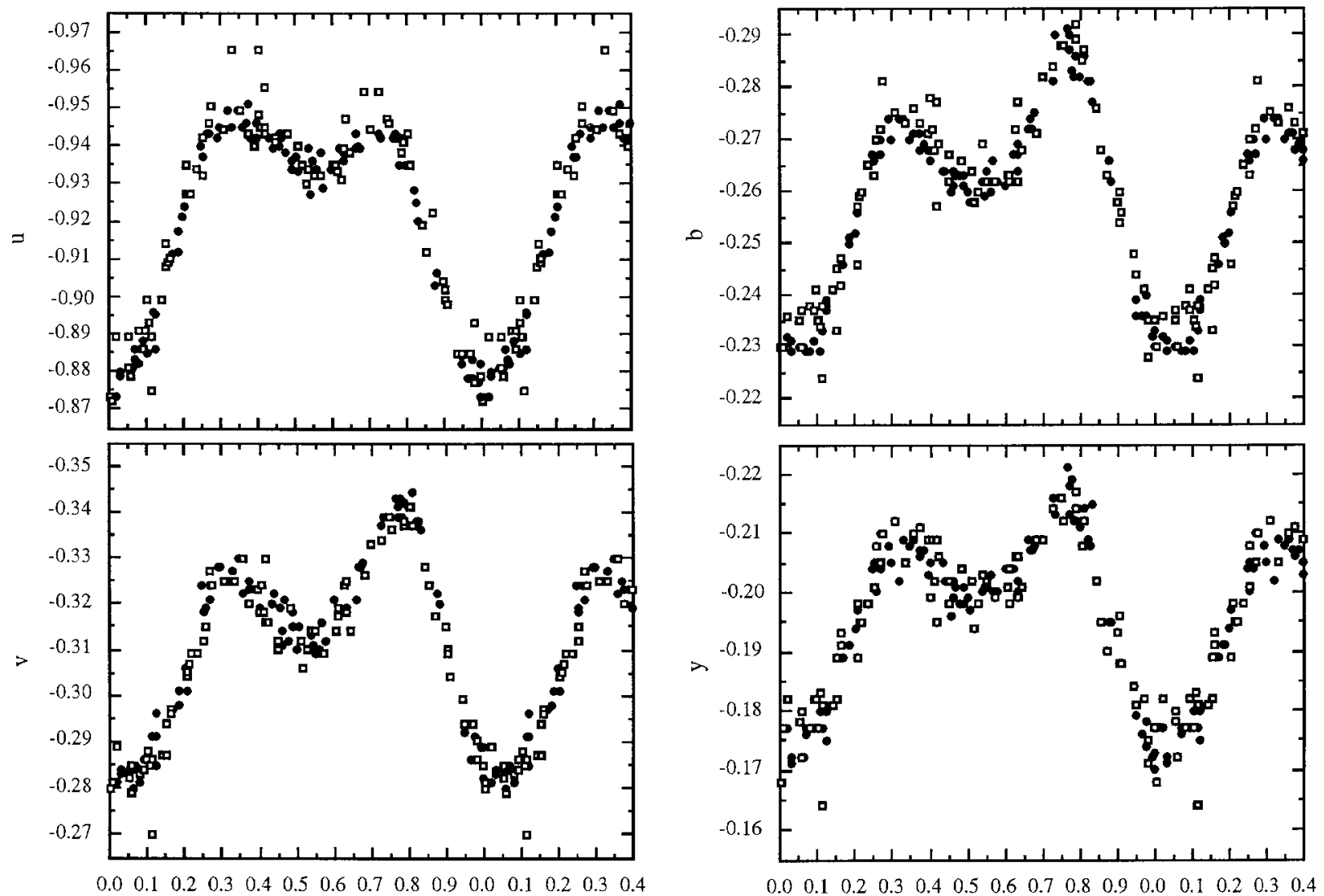

phase

Fig. 3. Differential FCAPT uvby photometry of HR 8434 (open squares from Adelman et al. 1994 and solid circles this paper) plotted with the ephemeris HJD $\left(y_{\text {min }}\right)=2441613.3990+1.43237 E$

As their light curves showed some differences with respect to those of Winzer (1974), it appeared worthwhile to obtain new observations which would also permit in principle a refinement of the period. Thus, 46 and 45 sets of additional new uvby differential photometry were obtained in the 1998-99 and 1999-2000 observing seasons, respectively. Comparison of the new with the older uvby data showed a slight phase shift. To bring both together requires a slight adjustment to the period and the zero point.

HJD $\left(y_{\min }\right)=2441613.3990 \pm 0.0002+1.43237 \pm$ $0.00003 E$.

No consideration was given to the data by Winzer (1974) as it consists of only a few values whose quality is not as good. The new observations form a smoother light curve which is better defined. To bring the two $u$ light curves into agreement required a shift of 0.02 mag. This star is somewhat Southerly for the FCAPT site. As the telescope was moved between the two sets of data, there may be discrepancies in the extinction correction. The light curves (Fig. 3) have slightly smaller amplitudes than previously found: $0.065 \mathrm{mag}$ in $u, 0.060 \mathrm{mag}$ in both $v$ and $b$, and $0.045 \mathrm{mag}$ in $y$. The light curves are all in phase. There appear to be slight differences in the old and new data sets near the primary minimum. Although the older values are more likely to be the problem, this indicates that another set of photometry should be taken in a few years. Note that the relative amplitude of primary maximum near phase 0.85 changes with bandpass with respect to the secondary maximum near phase 0.30 . This is indicative of nonuniform elemental abundance distributions across the stellar surface.

\section{Final comments}

Observations of the other 3 possibly variable Am stars found by Adelman (1998) if they also indicate constancy or binarity should settle the question of the class intrinsic variability for those stars outside of the variability strip. That HR 5341 is an intrinsically constant star suggests that current theories which suggest intrinsic constancy are correct.

The light curves of both HR 6967 and HR 8434 are examples what the FCAPT can do under favorable circumstances. For both the details of the light curves are evident. Additional observations in a few years can be used to both improve the accuracy of the period and resolve remaining discrepancies between data sets. The light curves 
of HD 142070 are not as nice. The amplitudes of variation are smaller, but still detectable. The results depend on the stellar declinations and the distances of the check and comparison stars from the variable. For HD 142070 we have a relatively Southerly star whose check and comparison stars are not as close to the variable as one would like. It is a star worth observing again either from a more Southerly observatory or using comparison and check stars closer to the variable.

Acknowledgements. This work was supported in part by NSF grant AST-9528506. I appreciate the continuing efforts of Louis J. Boyd, Robert J. Dukes, Jr., and George P. McCook to keep the FCAPT operating properly. I thank Pierre North for providing me with his unpublished Geneva photometry of HR 6967 which permitted me to give a more accurate ephemeris than I could with my data alone. This research has made use of the SIMBAD database, operated at the CDS, Strasbourg, France.

\section{References}

Adelman, S. J. 1998, A\&AS, 132, 93

Adelman, S. J., Brown, B. H., Caliskan, H., Reese, D. F., \& Adelman, C. J. 1994, A\&AS, 106, 333
Adelman, S. J., Pi, C.-L. M., \& Rayle, K. E. 1998, A\&AS, 133, 197

Adelman, S. J., Rayle, K. E., \& Pi, C.-L. M. 1999, A\&AS, 136, 379

Catanzaro, G., Leone, F., \& Catalano, F. A. 1999, A\&AS, 134, 211

ESA, 1997, The Hipparcos and Tycho Catalogs, SP-1200

Hoffleit, D. 1982, The Bright Star Catalogue, 4th edition (Yale University Observatory, New Haven, CT)

Hoffleit, D., Saladyga, M., \& Wlasuk, P. 1983, A Supplement to the Bright Star Catalogue (Yale University Observatory, New Haven, CT)

Horne, J. H., Baliunas, S. L. 1986, ApJ, 302, 757

Mathys, G., Hubrig, S., Landstreet, J. D., Lanz, T., \& Manfroid, J. 1997, A\&AS, 123, 353

Michaud, G., \& Proffitt, C. R. 1993, in ASP Conf. Proc. 44, Peculiar Versus Normal Phenomena in A-Type and Related Stars, ed. M. M. Dworetsky, F. Castelli, \& R. Faraggiana (ASP, San Francisco), 439

North, P. 1992, in Yu. V. Glagolevskij, I. I. Romanyuk, Proc. Int. Meeting on Problem Physics and Evolution of the Stars. Stellar Magnetism, NAUKA, Saint-Petersburg, 73

Roberts, D. H., Lehar, J., \& Dreher, J. W. 1987, AJ, 93, 968

Scargle, J. D. 1982, ApJ, 263, 835

Winzer, J. E. 1974, Ph.D. Thesis, University of Toronto 